\title{
Schema Provoke False Knowing Even When Schema-Consistent Targets Had Not Been Presented
}

\author{
Ryoma Yamada $^{1}$, Yukio Itsukushima ${ }^{2,3}$, Tanjeem Azad $^{3} \&$ D. Stephen Lindsay ${ }^{3}$ \\ ${ }^{1}$ Graduate School of Literature and Social Sciences, Nihon University, Tokyo, Japan \\ ${ }^{2}$ College of Humanities and Sciences, Nihon University, Tokyo, Japan \\ ${ }^{3}$ Department of Psychology, University of Victoria, Victoria, Canada \\ Correspondence: Ryoma Yamada, Graduate School of Literature and Social Sciences, Nihon University, 3-25-40 \\ Sakurajosui, Setagaya-ku, Tokyo 156-8550, Japan. E-mail: ryom_cc@yahoo.co.jp
}

Received: March 23, 2014

Accepted: June 30, $2014 \quad$ Online Published: July 30, 2014

doi:10.5539/ijps.v6n3p62

URL: http://dx.doi.org/10.5539/ijps.v6n3p62

\begin{abstract}
Human memory is not always an accurate record of experienced events. Information that has never been experienced but is consistent with a relevant schema is sometimes mistaken as memory, giving rise to false memories. In this study, we focused on whether schema can provoke false memory for actions and for objects even when schema-consistent targets had not been presented. We presented schema-inconsistent actions and schema-inconsistent objects in a slide sequence depicting a kitchen. Later, we administered an old/new recognition test with remember/know judgments and Perception/Thought/Emotion/Context ratings for schema-inconsistent targets, schema-consistent distracters, and schema-inconsistent distracters. Both for the actions and the objects, participants more often falsely recognized schema-consistent distracters than schema-inconsistent distracters. That is, memory can be reconstructed along the scene schema, provoking false memory. However, these false memories were not typically accompanied by "remember" judgments but rather by "know" judgments. The similarity between schema-consistent targets and schema-consistent distracters is an essential factor for false recollection.
\end{abstract}

Keywords: everyday scene, false memory, Perception/Thought/Emotion/Context questionnaire, remember/know judgment, schema

\section{Introduction}

\subsection{Previous Studies}

Humans organize memory into their knowledge for particular scenes or situations, what Bartlett (1932) termed "schema." A large number of previous studies reported that information that had not been experienced on a particular scene but is consistent with the schema of the scene is sometimes falsely remembered as having been experienced in that scene. When after studying a familiar scene participants are given a recognition memory test, they more often falsely recognized schema-consistent distracters than schema-inconsistent distracters (Graesser, Gordon, \& Sawyer, 1979; Graesser, Woll, Kowalski, \& Smith, 1980; Lampinen, Copeland, \& Neuschatz, 2001; Lampinen, Faries, Neuschatz, \& Toglia, 2000; Nakamura, Graesser, Zimmerman, \& Riha, 1985; Neuschatz, Lampinen, Preston, Hawkins, \& Toglia, 2002; Pezdek, Whetstone, Reynolds, Askari, \& Dougherty, 1989; Yamada \& Itsukushima, 2013a). In order to explain such false recognition for schema-consistent distracters, Graesser and his colleagues suggested the schema-copy-plus-tag model (Graesser et al., 1979; Graesser et al., 1980; Graesser, Kassler, Kreuz, \& McLain-Allen, 1998; Nakamura et al., 1985; Nakamura \& Graesser, 1985). The schema-copy-plus-tag model claims that when participants experience an event, they activate the schema of the event in order to comprehend the event. This leads them to elaborate schema-inconsistent targets in an explicit way because the schema clashes with these targets. In contrast, schema-consistent targets are unlikely to be elaborated. Furthermore, the model claims that memory is reconstructed based on the schema of the scene and that participants therefore have difficulty discriminating schema-consistent targets from schema-consistent distracters on the test.

A substantial number of published studies have explored the subjective phenomenology of false memories. Some of these studies used a remember/know judgment procedure (e.g., Payne, Elie, Blackwell, \& Neuschatz, 1996). 
The remember/know judgment was introduced by Tulving (1985) as a procedure to examining subjective experience, and developed by Gardiner (1988) and Rajaram $(1993,1996)$. In this procedure, participants are required to make "remember" judgment when they recollect the details for the recognized item, and to make "know" when the recognized item is familiar but no details of the studiy-list encounter with the item are recollected. Other studies have asked subjects to rate the characteristics of their memories on various dimensions, such as the extent to which they recollect perceptual details, their thoughts and emotions, and/or the context in which the item was recollected (Lampinen et al., 2000, Neuschatz et al., 2002): a Perception/Thought/Emotion/Context (PTEC, for short below) questionnaire, based on the Memory Characteristics Questionnaire (Johnson, Foley, Suengas, \& Raye, 1988). Many studies along these lines have found that false memories for schema-consistent distracters are sometimes accompanied by reports of recollection. The familiarity-plus-corroboration model (Lampinen et al., 2000; Lampinen, Meier, Arnal, \& Leding, 2005; Lampinen, Ryals, \& Smith, 2008) provides an explanation for such false recollection. When the distracter has high similarity with targets, participants feel strong familiarity for the distracter. This feeling of familiarity leads participants to search for details in memory that corroborate the distracter. Such searches are likely to retrieve memories of details of schema-consistent targets due to their similarity to the schema-consistent distracter that is being used as a search cue. Consequently, details or features of targets are likely to come to mind and to fit well with the person's schema-driven expectations and hence are sometimes misattributed to the to-be-remembered source ("content borrowing"; for related ideas, see Johnson, Hashtroudi, \& Lindsay, 1993; Lindsay, 2014).

\subsection{Purpose}

To sum up, in theory the schema of the scene makes the boundary ambiguous between schema-consistent target and schema-consistent distracter. If so, we had one question: whether schema-consistent distracters would be falsely recognized even when schema-consistent targets had not been presented in the event. We addressed that question by having participants study a series of slides depicting a man performing schema-inconsistent actions with schema-inconsistent objects in a kitchen scene. Of course, the kitchen scene included schema-consistent appointments (e.g., stove, sink, counter) but none of the actions he performed were schema-consistent, and none of the objects shown in the background were schema-consistent. If memory reconstruction based on the schema activated by the scene is a sufficient factor to provoke false memory, then participants would more often falsely recognized schema-consistent distracters than schema-inconsistent distracters despite the absence of scheme-consistent targets. If, in contrast, the similarity between schema-consistent targets and schema-consistent distracters is necessary to induce false memory, then participants would recognize schema-inconsistent distracters as often as schema-consistent distracters. We also assessed whether schema-consistent distracters would be recollected and which details would be falsely recollected when distracters were recognized.

Furthermore, in the present study, we compared memory performance for actions versus objects. Most of the previous studies confirmed false recognition and false recollection for schema-consistent distracters that were either action items (e.g., Lampinen et al., 2001) or object items (e.g., Neuschatz et al., 2002). Yamada and Itsukushima (2013a, 2013b) assessed the effect of schema for both action and object items. It was revealed that schema-consistent distracters were falsely recognized both for actions and objects, but that false recognition was more common for objects than for actions. Because Yamada and Itsukushima (2013a, 2013b) confirmed frequent recollection for schema-consistent action targets, they considered that recollecting schema-consistent action targets prevented false recognition for schema-consistent action distracters. Such a memory monitoring process whereby recollecting the target prevents false memory for related distracter is called "recollection rejection" (Brainerd \& Reyna, 2002; Lampinen et al., 2005; Odegard \& Lampinen, 2005; Rotello \& Heit, 1999). However, there is another monitoring process that may be used to reject distracters: the "distinctiveness heuristic." This is the memory monitoring process whereby the failure to recollect details that participants believe they would recollect leads them to reject that test probe (Gallo, Bell, Beier, \& Schacter, 2006; Schacter, Israel, \& Racine, 1999). It is possible that participants have lower expectations regarding recollections of objects than of actions, such that schema-consistent object distracters are more likely to be falsely recognized than are schema-consistent action distracters. In order to examine which monitoring process accounts for the observed pattern of less false memory for schema-consistent action than schema-consistent object, we compared recognition rates and subjective experiences between actions and objects. If even in the present study participants more frequently falsely recognize schema-consistent object distracters than schema-consistent action distracters, then that would suggest that the "distinctiveness heuristic" causes this phenomenon because only schema-inconsistent targets that do not relate to schema-consistent distracters are presented. 


\subsection{Research Design}

We presented schema-inconsistent action targets and schema-inconsistent object targets in a slide sequence depicting a kitchen. The kitchen included schema-consistent appointments (e.g., a stove, a counter, a sink), but all of the smaller objects in the scene were schema-inconsistent. Later we administered a recognition test that consisted of schema-inconsistent action and object targets, schema-consistent action and object distracters, and schema-inconsistent action and object distracters. The test used a remember/know judgment procedure, and a PTEC questionnaire.

\section{Method}

\subsection{Participants}

Twenty-nine undergraduate students from Nihon University (20 females and 9 males) participated in this experiment. The mean age was 19.8 years $(S D=0.9)$.

\subsection{Design}

A 2 (Consistency with Schema: Consistent, Inconsistent) $\times 2$ (Item: Action, Object) design was adopted. Both factors were varied within-participants. Because all presented targets were schema-inconsistent, only the latter factor was adopted for the analyses for memory performances for the targets.

\subsection{Materials}

We carried out a prior normative study to determine schema-inconsistent and schema-consistent items in the kitchen scene. Eleven graduate students from Nihon University ( 5 females and 6 males) who did not participate in the present experiment participated in the normative study. The mean age was 25.7 years $(S D=2.1)$. We showed them a list of 30 actions and 30 objects. The list was printed on four sheets in Japanese. We told participants to rate these items using a 7-point scale on which 1 denoted extremely schema-inconsistent and 7 denoted extremely schema-consistent. These ratings produced 10 items for each category of test items: schema-inconsistent action targets $(M=1.74, S D=0.5)$, schema-inconsistent object targets $(M=1.45, S D=0.4)$, schema-consistent action distracters $(M=6.75, S D=0.3)$, schema-consistent object distracters $(M=6.65, S D=$ $0.3)$, schema-inconsistent action distracters $(M=1.34, S D=0.4)$, and schema-inconsistent object distracters $(M$ $=1.35, S D=0.3)$. These items are listed in the Appendix. We conducted a one-way analysis of variance (ANOVA) on participants' mean ratings of the 6 items (Item Type: Schema-Inconsistent Action Target, Schema-Inconsistent Object Target, Schema-Consistent Action Distracter, Schema-Consistent Object Distracter, Schema-Inconsistent Action Distracter, Schema-Inconsistent Object Distracter). The main effect of Item Type was significant $\left(F(5,50)=783.41, p<.01, M S e=.10, \eta_{\mathrm{G}}{ }^{2}=.98\right)$. We applied comparisons of Holm that generally showed lower scores for the four schema-inconsistent items relative to the two schema-consistent items. The fact that we selected the items that were easy to take pictures as the target indirectly caused the result that schema-inconsistent action targets showed higher scores than did the other three schema-inconsistent items. However, we do not see this as a problem since the mean ratings for schema-inconsistent items were generally much lower than schema-consistent items.

We presented the targets in consecutive "flip slides", 1 slide per second, comprising a total of 50 slides. Throughout the slides a man in his thirties performed 10 schema-inconsistent actions, so that describing each action required 5 consecutive slides. In the background of the slides, 10 schema-inconsistent objects were shown. The man never touched the objects.

\subsection{Tasks}

The recognition test consisted of the previously studied 10 schema-inconsistent action targets and 10 schema-inconsistent object targets, 10 schema-consistent action distracters, 10 schema-consistent object distracters, 10 schema-inconsistent action distracters, and 10 schema-inconsistent object distracters. The test was printed in Japanese on four pages, with action items listed on two pages and object items appearing on the remaining two pages. The order of targets and distracters within each action and object type was randomized. Presentation order of action and object item pages was counterbalanced across participants.

The instructions for the remember/know judgment procedure were adopted from Rajaram (1993). When participants recognized an item, they were instructed to write "R" or "K." They wrote "R" if they consciously recollected the details of having studied item, or wrote " $\mathrm{K}$ " if they were certain of the item's presentation in the slides but did not experience an accompanying recollection.

Participants were provided with further instructions regarding the PTEC questionnaire. Following each remember judgment to test items, participants were instructed to write "P" if accompanying perceptual details 
were recollected with the item; " $\mathrm{T}$ " to indicate a recollection of a thought that accompanied the item's initial presentation; " $E$ " to indicate a re-experiencing of an emotion that accompanied the item's initial presentation; and " $\mathrm{C}$ " if accompanying contextual details were recollected with the item, such as the item's relationship with other items. Participants had the option to provide more than one $\mathrm{P}, \mathrm{T}, \mathrm{E}$, or $\mathrm{C}$ rating for each item marked as a remember judgment.

\subsection{Procedure}

Participants completed the experiment in groups of one to four in a testing room and each participant sat in front of a display (NEC: PX-61XM2). Participants were informed by the experimenter that they would be viewing a series of slides that described a kitchen. To encourage intentional learning, participants were instructed to carefully memorize the content and description of the slides for a later memory test. Following the instruction, the experimenter presented to participants the "flip slides" by controlling a computer (HP: ProBook 4520s) to show each slide for one second. Described slide resolution was $1011 \times 768$ pixels (circa $100 \times 76 \mathrm{~cm}$ ). Participants then completed a math activity for 10 minutes as part of a filler task. Following the filler task, the experimenter handed to participants five sheets of papers. The instructions for the old/new recognition test, the remember/know judgment test, and the PTEC questionnaire appeared on the first sheet and all instructions and test items were written in Japanese. Test items were then presented on the subsequent four pages. The experimenter read the instructions to participants. For the recognition test, participants were instructed to place a " $\circ$ " next to the items that were presented in the slides, and place a " $\times$ " next to the items that was not presented in the slides. Then participants started the test. At the end, participants were fully debriefed by the experimenter.

\section{Results}

\subsection{Data}

Table 1 shows the mean recognition rates of items (i.e., hit rates to schema-inconsistent action and object targets and false alarm rates to all of the distracters), remember/know and perception/thoughts/emotion/contextual judgments. These rates were calculated by dividing the number of times participants recognized or judged each item by the number of items (10) and then averaging.

Table 1. Mean proportion of recognized items $(N=29)$

\begin{tabular}{|c|c|c|c|c|c|c|c|}
\hline & $\begin{array}{l}\text { Overall } \\
M(S D)\end{array}$ & $\begin{array}{l}\text { Remember } \\
M(S D)\end{array}$ & $\begin{array}{l}\text { Know } \\
M(S D)\end{array}$ & $\begin{array}{l}\text { Perception } \\
M(S D)\end{array}$ & $\begin{array}{l}\text { Thought } \\
M(S D)\end{array}$ & $\begin{array}{l}\text { Emotion } \\
M(S D)\end{array}$ & $\begin{array}{l}\text { Context } \\
M(S D)\end{array}$ \\
\hline \multicolumn{8}{|l|}{$\begin{array}{l}\text { Target } \\
\text { action }\end{array}$} \\
\hline $\begin{array}{l}\text { schema-inconsistent } \\
\text { object }\end{array}$ & $.71(.17)$ & $.66(.22)$ & $.05(.12)$ & $.50(.32)$ & $.27(.27)$ & $.20(.18)$ & $.04(.10)$ \\
\hline schema-inconsistent & $.70(.20)$ & $.57(.23)$ & $.13(.17)$ & $.44(.25)$ & $.18(.19)$ & $.11(.15)$ & $.08(.16)$ \\
\hline \multicolumn{8}{|l|}{$\begin{array}{l}\text { Distracter } \\
\text { action }\end{array}$} \\
\hline schema-consistent & $.04(.08)$ & $.00(.02)$ & $.04(.08)$ & $.00(.02)$ & $.00(.02)$ & $.00(.00)$ & $.00(.00)$ \\
\hline $\begin{array}{l}\text { schema-inconsistent } \\
\text { object }\end{array}$ & $.01(.04)$ & $.00(.02)$ & $.01(.03)$ & $.00(.02)$ & $.00(.00)$ & $.00(.00)$ & $.00(.00)$ \\
\hline schema-consistent & $.16(.22)$ & $.01(.04)$ & $.15(.22)$ & $.01(.04)$ & $.00(.02)$ & $.00(.00)$ & $.00(.02)$ \\
\hline schema-inconsistent & $.05(.09)$ & $.01(.03)$ & $.04(.08)$ & $.01(.03)$ & $.00(.02)$ & $.00(.00)$ & $.00(.00)$ \\
\hline
\end{tabular}

\subsection{Data Analysis}

We conducted a paired $t$-test for the responses for the targets, and a 2 (Consistency with Schema of Distracter: Consistent, Inconsistent) $\times 2$ (Item: Action, Object) ANOVA for the responses for the distracters. When the interaction was significant, an analysis of simple main effects was performed. We did not analyzed "remember" judgments and PTEC judgments for the distracters because of the low rate of "remember" judgments to distracters. 


\subsection{Recognition Results}

There was no significant difference between the hit rate for schema-inconsistent action targets and schema-inconsistent object targets $(t(28)=0.24$, n.s., $p=.81, r=.03)$.

For the mean false alarm rates, ANOVA showed a significant main effect of Consistency with Schema of Distracter $\left(F(1,28)=9.07, p<.01, M S e=.02, \eta_{\mathrm{G}}{ }^{2}=.08\right)$ and a significant main effect of Item $(F(1,28)=12.34$, $\left.p<.01, M S e=.02, \eta_{\mathrm{G}}{ }^{2}=.10\right)$. The interaction between Consistency with Schema of Distracter and Item was significant $\left(F(1,28)=4.46, p<.05, M S e=.01, \eta_{\mathrm{G}}{ }^{2}=.03\right)$. The simple main effect of Consistency with Schema of Distracter was marginally significant for Action $\left(F(1,28)=3.12, p<.10, M S e=.00, \eta_{\mathrm{G}}{ }^{2}=.05\right)$ and significant for Object $\left(F(1,28)=7.65, p<.01, M S e=.02, \eta_{\mathrm{G}}{ }^{2}=.11\right)$. The simple main effect of Item was significant for Consistent distracter $\left(F(1,28)=9.99, p<.01, M S e=.02, \eta_{\mathrm{G}}{ }^{2}=.13\right)$ and marginally significant for Inconsistent distracter $\left(F(1,28)=4.05, p<.10, M S e=.01, \eta_{\mathrm{G}}{ }^{2}=.07\right)$.

\subsection{Remember/Know Judgment Results}

There was no significant difference between the mean "remember" judgment rate for schema-inconsistent action targets and schema-inconsistent object targets $(t(28)=1.61$, n.s., $p=.12, r=.18)$.

Participants made more "know" judgments for schema-inconsistent object targets than for schema-inconsistent action targets $(t(28)=3.19, p<.01, r=.24)$.

For the mean "know" judgment rates for false alarm responses to distracters, ANOVA showed a significant main effect of Consistency with Schema of Distracter $\left(F(1,28)=8.54, p<.01, M s e=.02, \eta_{\mathrm{G}}{ }^{2}=.07\right)$, and a significant main effect of Item $\left(F(1,28)=10.70, p<.01, M s e=.01, \eta_{\mathrm{G}}{ }^{2}=.08\right)$. The interaction between Consistency with Schema of Distracter and Item was significant $\left(F(1,28)=4.21, p<.05, M s e=.01, \eta_{\mathrm{G}}{ }^{2}=.03\right)$. The simple main effect of Consistency with Schema of Distracter was marginally significant for Action $(F(1,28)=3.12, p<.10$, $\left.M s e=.00, \eta_{\mathrm{G}}{ }^{2}=.05\right)$ and significant for $\operatorname{Object}\left(F(1,28)=7.23, p<.05, M s e=.02, \eta_{\mathrm{G}}{ }^{2}=.10\right)$. The simple main effect of Item was significant for Consistent distracter $\left(F(1,28)=8.38, p<.01\right.$, Mse $\left.=.02, \eta_{\mathrm{G}}{ }^{2}=.11\right)$ and marginally significant for Inconsistent distracter $\left(F(1,28)=3.87, p<.10\right.$, Mse $\left.=.00, \eta_{\mathrm{G}}{ }^{2}=.07\right)$.

\subsection{PTEC Questionnaire Results}

There was no significant difference between the mean "perception" judgment rate for schema-inconsistent action targets and that for schema-inconsistent object target $(t(28)=1.10$, n.s., $p=.28, r=.10)$. There was no significant difference between the mean "contextual information" judgment rate for schema-inconsistent action targets and that for schema-inconsistent object target $(t(28)=1.21, n . s ., p=.23, r=.14)$. Participants made more "thought" judgments $(t(28)=2.31, p<.05, r=.18)$ and "emotion" judgments $(t(28)=2.77, p<.01, r=.28)$ for schema-inconsistent action targets than for schema-inconsistent object targets.

\section{Discussion}

We sought to explore the role of schema-consistent and inconsistent distracters in the development of false recognition and false memory for a pictorial scene. Both for actions and objects, participants more often falsely recognized schema-consistent distracters than schema-inconsistent distracters. Even though schema-consistent targets were never presented in the slides, schematic reconstruction still occurred, albeit at fairly low false recognition rates. Presumaly the global layout and appointments of the scene (Oliva \& Torralba, 2007) caused schematic reconstruction for the memory. Therefore participants falsely accepted schema-consistent distracters. However, these false recognitions were not recollective, that is, they were rarely accompanied with "remember" judgment. Rather, false recognition claims for the distracters were mostly accompanied by false feelings of knowing that the distracters were part of the studied scene. Perhaps this is because presented schema-inconsistent targets did not have similarity to schema-consistent distracters so that participants could not feel strong familiarity for the distracters. Then they did not borrow the details of schema-inconsistent targets in order to corroborate false recognitions for schema-consistent distracters. To sum up, although schematic reconstruction based on the scene information is sufficient to provoke false recognition, it may not be sufficient to induce false recollection but only induce the feeling of the familiarity. The similarity between presented schema-consistent target and schema-consistent distracter is essential for false recollection. It is also possible that the discrepancy between the presented schema-inconsistent targets and the non-presented schema-consistent distracters in conjunction with the remember/know and PTEC judgments encouraged participants' to carefully monitor their recollective experiences and increased the saliency of not having "seen" the distracters. Further investigation is needed regarding this issue.

Notable to the current finding is that participants more often falsely recognized schema-consistent object distracters than schema-consistent action distracters. Yamada and Itsukushima (2013a, 2013b) obtained the same 
result and attributed their finding to the "recollection rejection" monitoring process whereby false memory for the distracter can be prevented by the recollection for related target (Brainerd \& Reyna, 2002; Lampinen et al., 2005; Odegard \& Lampinen, 2005). Further support for the possibility of "recollection rejection" was obtained by the result showing that schema-consistent action targets were more often recognized than schema-consistent object targets. However, in the present study, schema-consistent targets were never presented. Furthermore, schema-inconsistent action targets were as frequently recognized with "remember" judgments as schema-inconsistent object targets. Hence, the "recollection rejection" process likely does not account for the current findings in which false recognition was lower for schema-consistent action than schema-consistent object distracters. Rather, a likely explanation for the current findings may be attributable to the use of the "distinctiveness heuristic" process whereby the absence of distinctive recollection is taken as evidence that the item must not have been studied (Gallo et al., 2006; Schacter et al., 1999). This distinctiveness heuristic may be particularly relevant to action distracters if participants might expect action memory to be more recollective and distinctive than object memory. Although participants could feel the familiarity for schema-consistent action distracters owing to schematic memory reconstruction, they did not falsely accept these distracters because they expected that they would recollect action items. This possibility aligns with the generally lower false remember rates for schema-consistent action distracters.

The overall recognition rate for schema-inconsistent action targets was almost identical to that for schema-inconsistent object targets. However, in previous studies, schema-inconsistent action targets were not so frequently recognized when both schema-consistent action targets and schema-inconsistent action targets had been presented together (Graesser et al., 1979; Nakamura et al., 1985; Yamada \& Itsukushima, 2013a). In Yamada and Itsukushima (2013a), one possibility was raised for such cases; the elaboration for schema-inconsistent action target could be inhibited due to the attempt to comprehend the whole context of the scene. Comprehending the whole context of the scene can substantially depend on the capture of a sequence of schema-consistent actions. On this point, schema-inconsistent action targets can disturb the comprehension of the context of the scene. Hence, participants may inhibit the elaboration of schema-inconsistent action targets. However, in the present experiment, participants may not have comprehended the entire context of the scene or inhibited elaboration because all of the presented action targets were schema-inconsistent; this may have contributed to the saliency of these items and to the very low false recognition rates.

In conclusion, the present research shows that encoding schema-inconsistent targets regarding a scene is sufficient to provoke false recognition for non-presented schema-consistent distracter. The schematic reconstruction made participants feel the familiarity for schema-consistent distracter. However, the false recognition for schema-consistent distracters only prompted feeling of knowing than recollection. We speculate that the similarity between schema-consistent targets and schema-consistent distracters may be necessary to induce false recollective memories.

\section{Acknowledgments}

We give an address of thanks to Yayoi Kawasaki and Masaya Mochizuki for the comments, Kosuke Kikumori for assisting in making the "flip slides", and all participants.

This work was supported by MEXT Grant-in-Aid for Scientific Research on Innovative Areas Grant Number 23101006.

\section{References}

Bartlett, F. C. (1932). Remembering: A study in experimental and social psychology. Cambridge: Cambridge university press.

Brainerd, C. J., \& Reyna, V. F. (2002). Recollection rejection: How children edit their false memories. Developmental Psychology, 38, 156-172. http://dx.doi.org/10.1037/0012-1649.38.1.156

Gallo, D. A., Bell, D. M., Beier, J. S., \& Schacter, D. L. (2006). Two types of recollection-based monitoring in younger and older adults: Recall-to-reject and the distinctiveness heuristic. Memory, 14, 730-741. http://dx.doi.org/10.1080/09658210600648506

Gardiner, J. M. (1988). Functional aspects of recollective experience. Memory \& Cognition, 16, 309-313. http://dx.doi.org/10.3758/BF03197041

Graesser, A. C., Gordon, S. E., \& Sawyer, J. D. (1979). Recognition memory for typical and atypical actions in scripted activities: Test of a script pointer + tag hypothesis. Journal of Verbal Learning and Verbal Behavior, 18, 319-332. http://dx.doi.org/10.1016/S0022-5371(79)90182-8 
Graesser, A. C., Kassler, M. A., Kreuz, R. J., \& McLain-Allen, B. (1998). Verification of statements about story worlds that deviate from normal conceptions of time: What is true about Einstein's Dreams? Cognitive Psychology, 35, 246-301. http://dx.doi.org/10.1006/cogp.1998.0680

Graesser, A. C., Woll, S. B., Kowalski, D. J., \& Smith, D. A. (1980). Memory for typical and atypical actions in scripted activities. Journal of Experimental Psychology: Human Learning and Memory, 6, 503-515. http://dx.doi.org/10.1037/0278-7393.6.5.503

Johnson, M. K., Foley, M. A., Suengas, A. G., \& Raye, C. L. (1988). Phenomenal characteristics of memories for perceived and imagined autobiographical events. Journal of Experimental Psychology: General, 117, 371-376. http://dx.doi.org/10.1037/0096-3445.117.4.371

Johnson, M. K., Hashtroudi, S., \& Lindsay, D. S. (1993). Source monitoring. Psychological Bulletin, 114, 3-28. http://dx.doi.org/10.1037/0033-2909.114.1.3

Lampinen, J. M., Copeland, S. M., \& Neuschatz, J. S. (2001). Recollections of things schematic: Room schemas revisited. Journal of Experimental Psychology: Learning, Memory, and Cognition, 27, 1211-1222. http://dx.doi.org/10.1037/0278-7393.27.5.1211

Lampinen, J. M., Faries, J. M., Neuschatz, J. S., \& Toglia, M. P. (2000). Recollections of things schematic: The influence of scripts on recollective experience. Applied Cognitive Psychology, 14, 543-554. http://dx.doi.org/10.1002/1099-0720(200011/12)14:6<543::AID-ACP674>3.0.CO;2-K

Lampinen, J. M., Meier, C. R., Arnal, J. D., \& Leding, J. K. (2005). Compelling untruths: Content borrowing and vivid false memories. Journal of Experimental Psychology: Learning, Memory, and Cognition, 31, 954-963. http://dx.doi.org/10.1037/0278-7393.31.5.954

Lampinen, J. M., Ryals, D. B., \& Smith, K. (2008). Compelling untruths: The effect of retention interval on content borrowing and vivid false memories. Memory, 16, 149-156. http://dx.doi.org/10.1080/09658210701839277

Lindsay, D. S. (2014). Memory source monitoring applied. In T. J. Perfect, \& D. S. Lindsay (Eds.), The SAGE handbook of applied memory (pp. 59-75). London, UK: Sage. http://dx.doi.org/10.4135/9781446294703.n4

Nakamura, G. V., \& Graesser, A. C. (1985). Memory for script-typical and script-atypical actions: A reaction time study. Bulletin of the Psychonomic Society, 23, 384-386. http://dx.doi.org/10.3758/BF03330191

Nakamura, G. V., Graesser, A. C., Zimmerman, J. A., \& Riha, J. (1985). Script processing in a natural situation. Memory \& Cognition, 13, 140-144. http://dx.doi.org/10.3758/BF03197006

Neuschatz, J. S., Lampinen, J. M., Preston, E. L., Hawkins, E. R., \& Toglia, M. P. (2002). The effect of memory schemata on memory and the phenomenological experience of naturalistic situations. Applied Cognitive Psychology, 16, 687-708. http://dx.doi.org/10.1002/acp.824

Odegard, T. N., \& Lampinen, J. M. (2005). Recollection rejection: Gist cuing of verbatim memory. Memory \& Cognition, 33, 1422-1430. http://dx.doi.org/10.3758/BF03193375

Oliva, A., \& Torralba, A. (2007). The role of context in object recognition. Trends in Cognitive Sciences, 11, 520-527. http://dx.doi.org/10.1016/j.tics.2007.09.009

Payne, D. G., Elie, C. J., Blackwell, J. M., \& Neuschatz, J. S. (1996). Memory illusions: Recalling, recognizing, and recollecting events that never occurred. Journal of Memory and Language, 35, 261-285. http://dx.doi.org/10.1006/jmla.1996.0015

Pezdek, K., Whetstone, T., Reynolds, K., Askari, N., \& Dougherty, T. (1989). Memory for real-world scenes: The role of consistency with schema expectation. Journal of Experimental Psychology: Learning, Memory, and Cognition, 15, 587-595. http://dx.doi.org/10.1037/0278-7393.15.4.587

Rajaram, S. (1993). Remembering and knowing: Two means of access to the personal past. Memory \& Cognition, 21, 89-102. http://dx.doi.org/10.3758/BF03211168

Rajaram, S. (1996). Perceptual effects on remembering: Recollective processes in picture recognition memory. Journal of Experimental Psychology: Learning, Memory, and Cognition, 22, 365-377. http://dx.doi.org/10.1037/0278-7393.22.2.365

Rotello, C. M., \& Heit, E. (1999). Two-process models of recognition memory: Evidence for recall-to-reject? Journal of Memory and Language, 40, 432-453. http://dx.doi.org/10.1006/jmla.1998.2623 
Schacter, D. L., Israel, L., \& Racine, C. (1999). Suppressing false recognition in younger and older adults: The distinctiveness heuristic. Journal of Memory and Language, 40, 1-24. http://dx.doi.org/10.1006/jmla.1998.2611

Tulving, E. (1985). Memory and consciousness. Canadian Psychology, 26, 1-12. http://dx.doi.org/10.1037/h0080017

Yamada, R., \& Itsukushima, Y. (2013a). The effects of schema on recognition memories and subjective experiences for actions and objects. Japanese Psychological Research, 55, 366-377.

Yamada, R., \& Itsukushima, Y. (2013b). The schema provokes a disparity of false recollection between actions and objects in an everyday scene. Scandinavian Journal of Psychology, 54, 276-282. http://dx.doi.org/10.1111/sjop.12051

\section{Appendix A}

Schema-consistent items and schema-inconsistent items used in the experiment

\begin{tabular}{|c|c|c|}
\hline & action & object \\
\hline \multirow[t]{10}{*}{ schema-inconsistent target } & build a plastic model & binocular glasses \\
\hline & clean ear with an ear pick & castanet \\
\hline & look in a dictionary & clothes hanger \\
\hline & make a hole in a wood with a awl & clothes iron \\
\hline & pass a comb through hair & headphones \\
\hline & put a stamp on an envelope & microphone \\
\hline & raise an iron dumbbell & playing card \\
\hline & sew & saw \\
\hline & shave with a shaver & shoe horn \\
\hline & swing a bat & shovel \\
\hline \multirow[t]{10}{*}{ schema-consistent distracter } & boil a dish in a pot & bottle opener \\
\hline & control the frame on a range & chopping board \\
\hline & drain noodles & cooking chopsticks \\
\hline & heat the dish in a microwave oven & instant noodles \\
\hline & peel a vegetable with a peeler & kitchen sponge \\
\hline & place the dish on a plate & ladle \\
\hline & put a kettle over the fire & measuring spoon \\
\hline & set a kitchen timer & pepper pot \\
\hline & taste the dish & potholder \\
\hline & wrap the dish in an aluminum foil & toaster \\
\hline \multirow[t]{10}{*}{ schema-inconsistent distracter } & paint with a paintbrush on a drawing paper & butterfly net \\
\hline & fly a paper plane & hammer \\
\hline & play a guitar & helmet \\
\hline & polish a shoe & microscope \\
\hline & punch holes in papers with a puncher & computer mouse \\
\hline & roll a dice & sickle \\
\hline & sharpen a pencil with a pencil sharpener & stapler \\
\hline & shave a wood with a plane & tambourine \\
\hline & skip a rope & tie \\
\hline & swing a tennis racket & umbrella \\
\hline
\end{tabular}




\section{Copyrights}

Copyright for this article is retained by the author(s), with first publication rights granted to the journal.

This is an open-access article distributed under the terms and conditions of the Creative Commons Attribution license (http://creativecommons.org/licenses/by/3.0/). 